\title{
CHARACTERIZATION OF CONVERGENCE IN FUZZY TOPOLOGICAL SPACES
}

\author{
E. LOWEN \\ Department of Mathematics \\ Free University of Brussels \\ 1050 Brussels, Belgium

\section{R. LOWEN} \\ Dienst Wiskundige Analyse \\ University of Antwerp, R.U.C.A. \\ 2020 Antwerp, Belgium \\ (Received March 19, 1985)
}

ABSTRACT. In a fuzzy topology on a set $x$, the limit of a prefilter (i.e. a filter in the lattice $[0,1]^{X}$ ) is calculated from the fuzzy closure. In this way convergence is derived from a fuzzy topology. In ourpaper we start with any rule "lim" which to any prefilter $\$$ on $X$ assignz a function $\lim \$ \in[0,1]^{X}$. We give necessary and sufficient conditions for the function $\$ \rightarrow \lim 3$ in order that it can be derived from a fuzzy topology.

KEY WORDS AND PHRASES. Fuzzy topology, prefilter, convergence, diagonal operator. 1980 MATHEMATICS SUBJECT CLASSIFICATION CODE. 54A20, 54A40

\section{INTRODUCTION .}

The notion of convergence is one of the basic notions in analysis. Convergence can be described in any topological space, by means of nets or filters. In many concrete examples however, convergence is the primitive notion, and the topology, if such exists, is defined only afterwards. From this situation the need has grown to have an axiom system for convergence which makes it possible to recognize whether the convergence is topological. For net convergence such an axiom system was given in 1937 by Birkhoff [2], the crucial "topological" axiom being the iterated limit axiom. For filter convergence it was only in 1954 that Kowalsky [7] found a workable counterpart for this, the so-called diagonal condition for filter convergence. In the same paper he shows that if this condition is fulfilled then closures of sets are closed. Later one of the authors showed that then too adherences of filters are closed [8]. However the condition need not imply that the convergence is topological. The first axiom system completely describing topological convergence internally in terms of convergent filters was given by Cook and Fisher in 1967 [5]. The diag !l xndition again played a key role in the formulation of their solution. Since then this condition has proved to be a very useful notion in convergence theory, especially in theory of extensions [6], [7], [9], [19].

Since the introduction of the abstract notion of fuzzy topologies [10] in 1976 several concrete examples have shown that there too it is the notion of convergence 
which is paramount and which most clearly demonstrates the aim of fuzzy topology. See e.g. fuzzy topologies on hyperspaces of fuzzy sets [12], [13], on metric spaces [17] and on spaces of probability measures [15], [16]. In each of these cases the notion of convergence in the fuzzy topology permits to "measure" a "degree" with which a filter converges to a point, and in each case maximal degree of convergence is equivalent to classical topological convergence in some associated topology. In the examples jus: mentioned these are respectively the Hausdorff-Bourbaki hyperspace topology on closed sets [18], the metric topology, and the topology of weak convergence [1].

In the context of fuzzy topologies it is therefore equally important to characterize fuzzy topological convergence internally. In this paper we solve this question and give a set of 6 axioms which turn out to be necessary and sufficient for a fuzzy convergence to be fuzzy topological.

With regard to these axioms a number of comments are in order.

First, it turned out that the diagonal condition of Kowalsky cannot be translated in a straightforward manner. The classical condition of a filter being convergent to a point has no meaning and its substitute i.e. the information of the degree with which a filter converges to a point can only be handled analytically and thus has been incorporated as such in the diagonal condition. This "fuzzy" diagonal condition will play a key role in the characterization of convergence in fuzzy topological spaces. Second, the fundamental classical condition concerning the convergence of comparable filters has to be replaced by two separate axioms.

The first axiom is analogous to the classical one with the exception that only filters which in a certain sense are "horizontally" comparable may be considered. Due to the fact however that prime filters are not necessarily maximal we also have a type of "vertical" comparability for filters and we need a second axiom to deal with those. Finally, yet another axiom concerning the "overall" degree of convergence of a filter is required which is purely "fuzzy" in the sense that it has no classical meaning or counterpart.

At the end of our paper we restrict our 6 axioms to prime filters and show that these "prime versions" too already fully characterize fuzzy convergence. These prime versions are important because the convergence theory in fuzzy topological spaces is founded in large part on the use of prime filters.

2. PRELIMINARIES.

We recall that $I:=[0,1]$ and that if $X$ is a set $I^{X}$ denotes the set of all functions $X \rightarrow I$, i.e. all fuzzy sets on $X$, equipped with the usual lattice structure. As such $I^{X}$ is a complete and completely distributive lattice.

If $X$ is a set and $A \subset X$ then $1_{A}$ denotes the characteristic function of $A$ and if $A=\{x\}$ then we write $1_{x}$ for $1_{\{x\}}$.

In order to discern between filters on $X$ and a type of "fuzzy filters" we shall, for the latter, simply use the term introduced by Bourbaki [3] in the framework of general lattices.

A prefilter (resp. prime prefilter) (on $X$ ) is a prefilter (resp. prime prefilter) in the lattice $I^{X}$. If $u \in I^{X}$ then we denote by $\dot{\mu}$ the principal prefilter $\{\nu \mid \nu \geqslant \mu\}$.

The set $P(X)$ of all prefilters on $X$ is ordered by inclusion and fulfils analogous order properties as the set $\mathbf{F}(X)$ of all filters on $X$. We use the same well-known notations and terminology for both. 
Given $\mathcal{X} \in P(X)$, the set of all prime prefilters finer than $\mathcal{\nexists}$ was shown to have minimal elements [11] and this latter collection is denoted $P_{m}(\exists)$. Given $F \in \mathbf{F}(X)$ we shall then denote the set of all filters (resp. ultrafilters) finer than $F$ by $F(F)$ (resp. $\mathbf{U}(F)$ ). The following maps shall be required very often [11],

$$
\begin{aligned}
& \left.\left.\imath: P(X) \rightarrow \mathbf{F}(X): \exists \rightarrow\left\{\mu^{-1}\right] 0,1\right] \mid \mu \in \exists\right\} \\
& \omega: \mathbf{F}(X) \rightarrow P(X): F \rightarrow\left\{\mu \mid \exists F \in F: \mu \geqslant 1_{F}\right\} \\
& c: P(X) \rightarrow I: \nexists \rightarrow \inf _{\mu \in \mathcal{J}} \sup _{X \in X} \mu(x) \\
& c^{-}: P(x) \rightarrow I: \nexists \rightarrow \inf _{b \in P_{m}(\exists)} c(5)
\end{aligned}
$$

For any $\mathcal{B} \in P(X)$ we now further define

$$
2_{m}(\exists):=\{\omega(F) \vee \exists \mid F \supset\{(\exists)\} .
$$

PROPOSITION 2.1. The mapping

$$
\mathbf{F}(2(\exists)) \rightarrow 2_{m}(\exists): F \rightarrow \omega(F) \vee \exists
$$

is an order isomorphism and a V-lattice isomorphism. The image of $\mathbf{U}(\boldsymbol{l}(\exists)$ ) moreover coincides with $P_{m}(\exists)$.

PROOF. By construction the mapping is surjective and order preserving. Let $F_{i} \in \mathbf{F}(l(\exists)) \quad i=1,2$ and put $\exists_{i}:=\omega\left(F_{i}\right) \vee \exists$. Let $\exists_{1} \supset \exists_{2}$. If $F_{2} \in F_{2}$ then $1_{F_{2}} \in \mathcal{J}_{2}$ and thus there exist $\mu \in \mathcal{\exists}$ and $F_{1} \in F_{1}$ such that $1_{F_{2}}>1_{F_{2}} \wedge \mu$. This implies that $\left.\left.F_{2} \supset F_{1} \cap \mu^{-1}\right] 0,1\right]$ which together with $l(\exists) \subset F_{1}$ shows that $F_{2} \in F_{1}$. This at the same time shows that the mapping is injective and an order isomorphism.

Next let $F_{j} \in \mathbf{F}(\mathfrak{l}(\exists)$ ) for $j \in J$ then clearly

$$
\omega\left(\underset{j \in J}{V} F_{j}\right) \vee \exists \supset \underset{j \in J}{V} \omega\left(F_{j}\right) \vee \rightrightarrows
$$

Conversely if $F_{1} \in F_{j_{1}}, \ldots, F_{n} \in F_{j_{n}}$ for some $n \in \mathbb{N}_{0}$ and $\mu \in \mathcal{B}$ then clearly

$$
\left(\begin{array}{cc}
\Lambda & 1_{F_{j}}
\end{array}\right) \wedge \mu=\Lambda_{i=1}^{n}\left(1_{F_{i}} \wedge \mu\right) \in \underset{j \in J}{v} \omega\left(F_{j}\right) \vee \Im .
$$

The fact that the image of $\mathbf{U}\left(\mathfrak{l}(\exists)\right.$ ) is precisely $P_{m}(\exists)$ is nothing else than the result of Theorem $3.2[11]$. 口

REMARKS. $1^{\circ}$ In Theorem 3.2 [11] notation is slightly different. Note that $(\mathbb{Z}, U)$ there means $\omega(U) \vee \exists$ here. Also in [11] $\exists$ and $U$ are called compatible precisely if $u \supset \imath(\exists)$.

$2^{\circ}$ The mapping of Proposition 2.1 is not a lattice isomorphism as is seen taking $x$ arbitrary but such that $|x| \geqslant 2, F_{x}:=i_{x}$ for all $x \in x$ and $\mathcal{F}:=\{\mu \mid \inf \mu(x)>0\}$. Then it follows that

$$
\omega\left(\Lambda_{x \in X} F_{x}\right) \vee \exists=\exists
$$

whereas 


$$
\Lambda_{x \in X} \omega\left(F_{x}\right) \vee \exists=\{\mu \mid \mu(x)>0 \quad \forall x \in x\}
$$

$3^{\circ}$ Without further mention and whenever convenient we shall use the fact that $P_{m}(\exists)=\{\omega(U) \vee \exists \mid u$ ultra, $u \supset \imath(\exists)\}$.

For the sake of completiness and because it shall frequently be used in the sequel we recall the following result, however without proof.

It was proved implicitly in the proof of Theorem 6.2 [11] and explicitly as Lemmas 3.1 and 3.2 in $[14]$.

PROPOSITION 2.2. The following hold :

$1^{\circ}$ If $F$ is a filter on $X$ and for each $U \in \mathbf{U}(F): U_{U} \in U$ then there exists a finite subset $\mathbf{U}_{0} \subset \mathbf{U}(F)$ such that $\underset{\text { Ueu }}{U} U_{U} \in F$.

$2^{\circ}$ If $\exists$ is a prefilter and for each $\mathscr{H} \in P_{\mathrm{m}}(\exists): \mu_{\mathscr{H}} \in \mathscr{G}$ then there exists a finite subfamily $P_{0} \subset P_{m}(\exists)$ such that $\sup _{\mathscr{G} \in P_{0}} \mu_{\mathscr{G}} \in \mathcal{B}$.

PROPOSITION 2.3. The following hold :

$1^{\circ} l$ and $\omega$ are respectively an isotone surjection and injection

$2^{\circ}$ low $=i d_{\mathbf{F}}(x)$, wo,$\leqslant i d_{P(X)}$

$3^{c} c(\exists)=\sup _{\left(5 \in P_{m}(\exists)\right.} c(\mathbb{5})$

$4^{\circ} \rightrightarrows$ prime $\Rightarrow c(\exists)=c^{-}(\exists)$.

PROOF. $1^{\circ}$ and $2^{\circ}$ are analogous to similar statements in [11] and we shall leave this to the reader.

$3^{\circ}$ Actually more can be said : there exists $\mathbb{G} \in P_{m}(\exists)$ such that $c(\mathbb{J})=c(\exists)$. Indeed consider the filter

$$
\left.\left.F:=\left[\left\{\mu^{-1}\right] \alpha, 1\right] \mid \alpha<c(\exists)\right\}\right]
$$

then clearly $F \supset \imath(\exists)$. Now let $U$ be any ultrafilter finer than $F$ then $U \in \mathbf{U}(\imath(\mathcal{I})$ ) and since for all $\alpha<c(\exists)$ and $\left.\left.\mu \in \mathcal{\exists}: \mu^{-1}\right] \alpha, 1\right] \in U$ it follows that

$$
c(\omega(u) \vee \exists)=\inf _{\substack{u \in U \\ \mu \in \exists}} \sup _{x \in U} \mu(x) \geqslant c(\exists)
$$

while the other inequality is trivial.

$4^{\circ}$ Immediate from the definitions.

PROPOSITION 2.4. If $\exists$ is a prefilter and $\mathcal{H} \in \mathcal{Z}_{\mathrm{m}}(\exists)$ then $P_{\mathrm{m}}(\nVdash) \subset P_{\mathrm{m}}(\exists)$.

PROOF. Indeed, by Proposition 2.1 we can choose $H \in \mathbf{F}(l(\exists))$ such that $\mathcal{H}=\omega(H) \vee \exists$ and if $\mathscr{H} \in P_{m}(\mathcal{H})$ we can find $u \in \mathbf{U}(\mathfrak{l}(\mathcal{H}())$ such that $\mathscr{G}=\omega(U) \vee \mathcal{H}$. From $u \supset l(\mathcal{H}()=H$ we then have

$$
\begin{aligned}
\mathscr{G} & =\omega(U) \vee \mathfrak{H} \\
& =\omega(U) \vee \omega(H) \vee \Im \\
& =\omega(U) \vee \Im
\end{aligned}
$$

which together with the fact that $u \supset l\left(\mathcal{H}() \supset l(\exists)\right.$ proves that $\left(5 \in P_{m}(\exists)\right.$.

PROPOSITION 2.5. For any ultrafilter $U$, the fiber $l^{-1}(\{U\})$ is a chain.

PROOF. Let $\mathbb{G}, \mathfrak{G}, \in \mathrm{I}^{-1}(\{U\})$ and suppose that there exist $\mu \in \mathscr{G} \backslash \mathfrak{G}$, and $\mu^{\prime} \in \mathbb{6}, \backslash \mathbb{6}$. Now let 


$$
\begin{aligned}
& A:=\left\{x \mid \mu(x) \geqslant \mu^{\prime}(x)\right\} \\
& B:=\left\{x \mid \mu^{\prime}(x) \geqslant \mu(x)\right\}
\end{aligned}
$$

and suppose for instance that $A \in U$, then $1_{A} \in \mathcal{G}^{\prime}$ and thus ${ }^{1} A \mu^{\prime} \in \mathcal{G}^{\prime}$. However since $\mu \geqslant 1_{A} \wedge \mu^{\prime}$ this provides us with a contradiction. $\square$ PROPOSITION 2.6. If $\left(\exists_{i}\right)_{i=1}^{n}$ are prefilters then

$$
P_{m}\left(\bigcap_{i=1}^{n} \exists i\right) \subset \bigcup_{i=1}^{n} P_{m}\left(\Im_{i}\right)
$$

PROOF. If $\mathscr{G} \in P_{m}\left(\bigcap_{i=1}^{n} \exists i\right)$ and for all $i=1, \ldots, n$ we have $\mathscr{G} \supset \mathcal{J}_{i}$ then we can find $\nu_{i} \in \exists \mathbb{J}_{i} \backslash \mathfrak{G} i=1, \ldots, n$. Then however $\sup _{i=1}^{n} \nu_{i} \in \mathbb{G}$ which is a contradiction. Consequently there exist $i_{0} \in\{1, \ldots, n\}$ such that $\mathscr{G} \supset \exists_{i_{0}}$. That $\mathscr{G}$ is minimal is clear. a

We shall now introduce the so-called diagonal prefilter, a concept which generalizes the analogous notion introduced by Kowalsky [7].

DEFINITION 2.1. Given a collection of prefilters $\left(\exists_{j}\right)_{j \in J}$ on $X$ and a filter $A$ on $J$ we define and denote the diagonal prefilter (of the family $\left(\exists_{j}\right)_{j \in J}$ with respect to A) as

$$
\text { (11) }\left(\left(\mathcal{F}_{j}\right)_{j \in J}, A\right):=\operatorname{v}_{A \in A} \cap \bigoplus_{j \in A}
$$

$\therefore$ wing the easy verification that this is indeed a prefilter to the reader.

Since we shall often use this concept we now give some of its basic properties.

PROPOSITION 2.7. Let $\left(\mathcal{F}_{j}{ }^{\text {) }}{ }_{j \in J}\right.$ be a family of prefilters on $\mathrm{X}$ and $A$ a filter on $J$ then the following properties hold :

$1^{\circ}$ (1) $\left.\left(\mathcal{B}_{j}\right)_{j \in J}, A\right)=\bigcup_{A \in A} \cap_{j \in A} \exists_{j}$

$2^{\circ}$ If $\left(A_{\ell}\right)_{l \in L}$ is a family of filters on $J$ such that $A=\underset{l \in L}{\cap} A_{l}$ then

$$
\text { (11) }\left(\left(\mathcal{J}_{j}\right)_{j \in J}, A\right)=\bigcap_{l \in L} \text { (II) }\left(\left(\mathbb{F}_{j}\right)_{j \in J}, A_{l}\right)
$$

3० (1) $\left(\left(\mathcal{\exists}_{j}\right)_{j \in J}, A\right)=\left(\mathscr{C}_{j}\right)_{j \in J} \in \prod_{j \in J} P_{m}\left(\exists_{j}\right)$ (D) $\left(\left(\mathscr{C}_{j}\right)_{j \in J}, A\right)$

$4^{\circ}$ If each $\exists, j \in J$ is prime and $A$ is an ultrafilter then $\left.\operatorname{D}\left(\mathcal{I}_{j}\right)_{j \in J}, A\right)$ is prime.

PROOF. $1 \circ$ If $\mu \in \mathbb{( 1 )}\left(\left(\mathcal{\exists}_{j}\right)_{j \in J}, A\right), A_{1}, \ldots, A_{n} \in A$ and $\nu_{i} \in \cap_{j \in A_{j}} \mathcal{\exists}_{j} i=1, \ldots, n$ are such that $\nu_{1} \wedge \ldots \wedge \nu_{n} \leqslant \mu$ then putting $A:=A_{1} \cap \ldots \cap A_{n} i$ t follows that $\mu \in A_{j \in A} \mathcal{J}_{j}$.

$2^{\circ}$ One inclusion is trivial, to show the other one let us suppose

$\left.\nu \in \cap_{l \in L}(1)\left((\exists)_{j}\right)_{j \in J}, A_{\ell}\right)$. Then making use of $1^{\circ}$ it follows that for all $\ell \in L$ there exists $A_{\ell} \in A_{l}$ such that $\nu \in \underset{j \in A_{\ell}}{\cap} \exists_{\ell}$. If we let $A=\underset{l \in L}{U} A_{l}$ then $A \in A$ and $\nu \in \cap \bigoplus_{\ell \in A}$.

$3^{\circ}$ Again one inclusion is quite clear, to show the other one let $\left.v \notin \mathbb{( 1 )}\left(\mathcal{J}_{j}\right)_{j \in J}, A\right)$ and for each $j \in J$, if $\nu \notin \exists$, choose $\mathscr{G}_{j} \in P_{m}\left(\exists_{j}\right)$ such that $\nu \notin G_{j}$ whereas if $\nu \in \exists_{j}$ choose $\mathscr{G}_{j} \in P_{m}\left(\oiint_{j}\right)$ arbitrarily. Now by supposition and upon once again applying $1^{\circ}$, for each $A \in A$ there is $j \in A$ such that $\nu \notin \exists_{j}$ and thus $\nu \notin \mathbb{G}_{j}$. Consequently $v \notin(1)\left(\left(\mathbb{C}_{j}\right)_{j \in J}, A\right)$ and we are done.

$4^{\circ}$ Let $\mu v \nu \in \mathbb{D}\left(\left(\exists_{j}\right)_{j \in J}, A\right)$ and let $A \in A$ be such that $\mu v \nu \in \underset{j \in A}{\cap} \oiint_{j}$. 
If we put

$$
\begin{aligned}
& A_{1}:=\left\{j \in A \mid \mu \in \mathcal{J}_{j}\right\} \\
& A_{2}:=\left\{j \in A \mid \nu \in \mathcal{J}_{j}\right\}
\end{aligned}
$$

then f.i. $A_{1} \in A, \mu \in \underset{j \in A_{1}}{\cap} \mathcal{\exists}$ and we are done.

PROPOSITION 2.8. If $\left(G_{j}\right)_{j \in J}$ is a family of prime prefilters, $A$ is a filter on $J$

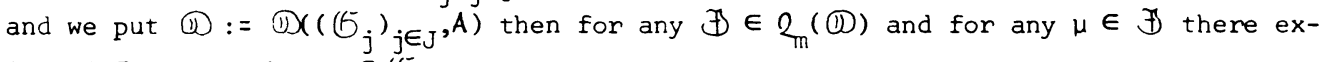
ists $j \in J$ such that $\mu \in G_{j}$.

PROOF. Let $F \in \mathbf{F}(l($ (I) $)), F \in F$ and $\mu \in \mathbb{D}$ and suppose that for all $j \in J$ we have $1_{F} \wedge \mu \notin 6_{j}$. From Proposition 2.7.10 we obtain that there exists $A \in A$ such that $\mu \in \mathscr{C}_{j}$ for all $j \in A$. Since $\mu=\left(1_{F} \wedge \mu\right) \vee\left(1_{F} \wedge \mu\right)$ it follows from our hypothesis that $1_{F^{c}} \wedge \mu \in \mathscr{G}_{j}$ for all $j \in A$ and consequently that $1_{F^{c}} \wedge \mu \in(1)$. Since

(1) $\subset \omega(F) \vee(1)$ this contradicts the fact that $1_{F} \wedge \mu \in \omega(F) \vee(1)$ and we are done.

We shall now turn to some preliminary results concerning convergence in fuzzy topological spaces.

W. recall that one of the equivalent ways of introducing a fuzzy topology on a set $X$ i. by means of the so-called fuzzy closure operator [10]. A fuzzy closure operator, b; tefinition, is a map ${ }^{-}: I^{X} \rightarrow I^{X}$ fulfilling
(1) $\bar{\alpha}=\alpha$
$\forall \alpha$ constant
(2) $\mu \geqslant \mu$
$\forall \mu \in I^{X}$
(3) $\overline{\mu v v}=\bar{\mu} v \bar{v}$
$\forall \mu, \nu \in I^{X}$
(4) $\bar{\mu}=\mu$
$\forall \mu \in I^{X}$

In this paper it is exclusively this definition of a fuzzy topology which we shall use. Now if $X$ is endowed with a fuzzy closure, i.e. is a fuzzy topological space, then convergence of prefilters is defined in the following way [11]. Adherence and limit are defined respectively as the mappings $P(X) \rightarrow I^{X}$ determined by

$$
\begin{aligned}
\operatorname{adh} \exists & :=\inf _{\mu \in \mathcal{H}} \bar{\mu} \\
\lim \exists: & =\inf _{\mathscr{G} \in P_{m}(\exists)} \operatorname{adh} \mathbb{G}
\end{aligned}
$$

The idea being that adh $\exists$ and $\lim \exists$ generalize respectively the set of adherence- and limitpoints of a filter.

We recall the most important properties which we shall require in the sequel, referring however to [11] for proofs.

PROPOSITION 2.9. If $X$ is a fuzzy topological space then the following hold for all $\exists, \mathbb{L} \in P(X):$

$1 \circ \exists \supset \mathbb{C} \Rightarrow \operatorname{adh} \Im \leqslant \operatorname{adh}(5$

$2^{\circ} \lim \rightarrow \leqslant \operatorname{adh} \circlearrowleft$

$3^{\circ}$ adh $\exists \leqslant c(\Im)$

$4^{\circ} \exists$ prime $\Rightarrow \lim \exists=\operatorname{adh} \exists$.

Because of its importance in our further considerations we display also the next result.

PROPOSITION 2.10. If $X$ is a fuzzy topological space then for any prefilter $\exists \in P(X)$

$$
\operatorname{adh} \exists=\sup _{\mathscr{G} \in P_{m}(\exists)} \operatorname{adh} \mathscr{W} \text {. }
$$


PROOF. This was shown implicitly in the proof of Theorem 2.6 [11].

PROPOSITION 2.11. If $X$ is a fuzzy topological space then for any prefilter $\exists \in P(X)$

$$
\operatorname{adh} \exists=\sup _{\mathcal{H} \supset} \lim \exists=\sup _{\mathcal{H} \supset \exists} \text { adh } \exists
$$

PROOF. Frum Propositions 2.9 and 2.10 we obtain

$$
\begin{aligned}
& \operatorname{adh} \exists=\sup _{\left(\zeta \in P_{m}(\Im)\right.} \lim (\zeta \\
& \leqslant \sup _{\exists \supset \exists} \lim \exists \\
& \leqslant \sup _{\mathfrak{H} \supset \exists} \text { adh } \nVdash \\
& \leqslant \text { adh } \oiint \text {. }
\end{aligned}
$$

We shall also require the following result.

PROPOSITION 2.12. If $X$ is a fuzzy topological space and $\rightarrow$ a prefilter on $X$ then for each $x \in X$ there exists $\mathscr{G} \in P_{m}(\exists)$ such that $\lim \mathscr{G}(x)=\operatorname{adh} \ni(x)$.

PROOF. Suppose on the contrary that for each $U \in \mathbf{U}(l(\exists))$ we have

$$
\lim (\omega(U) \vee \exists)(x)<\operatorname{adh} \exists(x),
$$

then for each $u \in U(u(\exists))$ we can find $U_{U} \in U$ and $\mu_{u} \in \exists$ such that

$$
\overline{1_{U} \wedge \mu_{U}}(x)<\operatorname{adh} \exists(x) \text {. }
$$

By Proposition 2.2 there exist $u_{1}, \ldots, u_{n} \in \mathbf{U}(\imath(\exists))$ and $U_{i}:=U_{u_{i}} \in u_{i}, i=1, \ldots, n$ such that $\bigcup_{i=1}^{n} U_{i} \in l(\exists)$. Let $\mu_{i}:=\mu_{u_{i}}, i=1, \ldots, n$ be the corresponding elements in $\exists$ and let

then we obtain

$$
\mu:=\inf _{i=1}^{n} \mu_{i}
$$

$$
\begin{aligned}
\underbrace{n}_{i=1} U_{i} & \bar{n} \\
& <\sup _{i=1} 1_{U_{i}} \wedge \mu_{i}(x) \\
& \sup _{i=1} \overline{1_{U} \wedge \mu_{i}}(x) \\
& <\operatorname{adh} \exists(x)
\end{aligned}
$$

which from the obvious fact that $1_{n} \wedge \mu \in \exists$ is a contradiction. 口

$$
\bigcup_{i=1}^{n} U_{i}
$$

As an immediate consequence remark that if $\mu \in I^{X}$ then for each $x \in X$ we can thus find $\mathscr{G} \in P_{m}(\dot{\mu})$ such that $\bar{\mu}(x)=\lim \mathscr{G}(x)$.

Finally, we introduce also the following concept which shall be of crucial importance in our considerations, since it is precisely the tool which permits to generalize Kowalsky's diagonal condition.

If $(S(y))_{y \in X}$ is a family of prefilters indexed by $x$-called a selection of prefiltersthen we define $\rho_{S}$ as the map

$$
\rho_{\mathcal{S}}: X \rightarrow I: x \rightarrow \lim S(x)(x) .
$$


The function (or fuzzy set) $\rho_{S}$ measures at each point the degree that $S(x)$ converges to $x$.

3. NECESSITY.

THEOREM 3.1 If $X$ is a fuzzy topological space then the map $P(X) \rightarrow I^{X}: \exists \rightarrow \lim \exists$ satisfies the following properties

(F1) For any prefilter $\exists: \lim \exists<\mathrm{c}^{-}(\exists)$

(F2) For any prefilters $¥, 36$ :

$$
\exists \subset \mathcal{H}, 2(\exists)=\imath(\mathcal{H}) \Rightarrow \lim \mathfrak{Z}<\lim \exists
$$

(C1) For any $\alpha \in I \backslash 0$ and $x \in X: \lim \alpha i_{x}>\alpha 1_{x}$

(C2) For any prefilter $\exists$ :

$$
\mathcal{H} \in 2_{m}(\exists) \Rightarrow \lim \exists<\lim \mathcal{H}
$$

(Cc) For any collection of prefilters $\left(\mathbb{B}_{j}\right)_{j \in J}$ and any filter $A$ on $J$ :

$$
\lim (1)\left(\left(\Im_{j}\right)_{j \in J}, A\right)>\inf _{j \in J} \lim \Im_{j}
$$

(cd) For any prefilter $\rightarrow$ and any selection of prefilters $(S(y))_{y \in x}$ such that $\rho_{S} \in \exists$ :

$$
\lim (1)\left((S(y))_{y \in x}, i(\exists)\right)>\lim \exists
$$
and moreover the map $I^{X} \rightarrow I^{X}: \mu \rightarrow \sup _{\left(\mathcal{G} \in P_{m}(\dot{\mu})\right.} \lim \mathscr{G}$ coincides with the original closure
on $X$.

PROOF. (F1) In case $\rightrightarrows$ is prime we have $c^{-}(\exists)=c(\exists)$ and

$$
\begin{aligned}
\lim \exists & =\inf _{\mu \in \mathcal{H}} \bar{\mu} \\
& <\inf _{\mu \in \Im} \sup _{x \in X} \mu(x) \\
& =c(\exists)
\end{aligned}
$$

In case $\mathcal{B}$ is arbitrary we deduce herefrom

$$
\begin{aligned}
\lim \exists & <\inf _{\mathscr{b} \in P_{m}(\exists)} \lim \mathscr{G} \\
& <\inf _{\mathfrak{G} \in P_{m}(\Im)} c(\mathscr{G}) \\
& =c^{-}(\exists)
\end{aligned}
$$

(F2) Let $\exists$ and $\mathcal{F}^{(l}$ be as postulated then we have

$$
\begin{aligned}
& \lim \mathcal{\exists}=\inf _{u \in U(\mathcal{H}(\mathcal{H}))} \lim \omega(u) \vee \mathfrak{H} \\
& <\inf _{\operatorname{u\in U}(\imath(\boxplus))} \lim \omega(U) \vee \Im \\
& =\lim \nexists
\end{aligned}
$$

(c1) Since $\alpha i_{x}$ is prime we have

$$
\begin{aligned}
\lim \alpha i_{x} & =\overline{\alpha 1_{x}} \\
& >\alpha 1_{x}
\end{aligned}
$$

(c2) Let $\exists$ and $\exists\left(\in \in Z_{m}(\exists)\right.$ be arbitrary then by Proposition 2.4 we immediately obtain 


$$
\begin{aligned}
\lim \exists & =\inf _{\mathscr{G} \in P_{\mathrm{m}}(\exists)} \lim \mathscr{\zeta} \\
& \leqslant \inf _{\mathscr{\zeta} \in P_{\mathrm{m}}(\mathcal{H})} \lim \mathscr{\zeta} \\
& =\lim \mathcal{F}(
\end{aligned}
$$

(Cc) In case each $\exists_{j}, j \in J$, is prime, using Proposition 2.8, and letting (1) $:=(1)\left(\left(\oiint_{j}\right)_{j \in J}, A\right)$ we obtain

$$
\begin{aligned}
& \lim (D)=\inf _{\mathscr{E} \in P_{m}(\text { (I) })} \inf _{\mu \in \mathcal{G}} \bar{\mu} \\
& \geqslant \inf \text { inf } \bar{\mu} \\
& j \in J \quad \mu \in \mathcal{F}_{j} \\
& =\inf _{j \in J} \lim \exists_{j} .
\end{aligned}
$$

In the general case, for each $j \in J$, let

$$
P_{m}\left(J_{j}\right)=\left\{\mathscr{G}_{k} \mid k \in k_{j}\right\}
$$

where the collections $K_{j}$ are chosen to be pairwise disjoint. For $A \subset J$ let

$$
A^{\prime}:=\bigcup_{j \in A}^{U} K_{j}
$$

then $\left\{A^{\prime} \mid A \in A\right\}$ is a filterbase on $J^{\prime}$. Let us denote the filter thus generated by $A^{\prime}$ Then from the first part of the proof it follows that

$$
\begin{aligned}
\lim & \text { (1) }\left(\left(G_{k^{\prime}}\right)_{k \in J^{\prime}}, A^{\prime}\right) \\
\geqslant & \inf _{k \in J^{\prime}} \lim \mathscr{G}_{k} \\
& \geqslant \inf _{j \in J} \lim \Im_{j}
\end{aligned}
$$

Following the straightforward verification that

$$
\text { (1) }\left(\left(\exists_{j}\right)_{j \in J}, A\right)=(1)\left(\left(G_{k}\right)_{k \in J}, A^{\prime}\right)
$$

we are done.

(cd) Let (1) $:=(1)\left((S(y))_{y \in X}, l(\exists)\right)$.

ASSERTION. The result holds if all $S(y), y \in X$ and $\rightarrow$ are prime.

Indeed, let $\mu \in(1)$. Then by Proposition 2.7.10 there exists $F \in l(\exists)$ such that $\mu \in \cap_{y \in F} S(y)$. Let

$$
\varphi:=1_{F} \wedge \rho_{S}
$$

then $\varphi \in \exists$. Further straightforward verification shows that

$$
\sup _{\left.\left.y \in \varphi^{-1}\right] 0,1\right]} \lim S(y) \geqslant \varphi
$$

and consequently sup $\lim S(y) \in \exists$.

$$
\left.\left.y \in p^{-1}\right] 0,1\right]
$$

Finally too by Proposition 2.9

$$
\begin{aligned}
& \bar{\mu} \geqslant \operatorname{adh} \underset{y \in F}{\cap} S(y) \\
& \left.\left.\geqslant \operatorname{adh} \operatorname{Sap}^{-1}\right] 0,1\right] \\
& \geqslant \sup _{\left.\left.y \in \varphi^{-1}\right] 0,1\right]} \lim S(y)
\end{aligned}
$$


which in turn implies $\bar{\mu} \in \supsetneqq$.

Consequently $\bar{\mu}=\bar{\mu}>\lim \ni$ which by the arbitrariness of $\mu \in(1)$ and by Proposition 2.9 shows that $\lim ($ ) $)>\lim ¥$.

ASSERTION. The result holds in general.

Let $(S(y))_{y \in x}$ and $\rightarrow$ be arbitrary prefilters, such that $\rho_{S} \in \supsetneqq$. First observe that for any selection $R:=(R(y))_{y \in X} \in \prod_{y \in X} P_{m}(S(y))$ we have

$$
\rho_{\mathfrak{S}}(y)=\lim S(y)(y)<\lim R(y)(y)=\rho_{\mathbb{R}}(y)
$$

and this for all $y \in X$. Consequently $\rho_{\mathbb{R}} \in \exists$ and a fortiori $\rho_{\mathbb{R}} \in \mathbb{G}$ for any $\left(5 \in P_{m}(\exists)\right.$.

From the first assertion we then already obtain that

$$
\lim (1)\left((\Omega(y))_{y \in X}, l((5))\right)>\lim (5) \text {. }
$$

Next observe that by Proposition 2.7.2 and $3^{\circ}$ we have

$$
\begin{aligned}
& \text { (1) }\left((S(y))_{y \in x}, l(\exists)\right)
\end{aligned}
$$

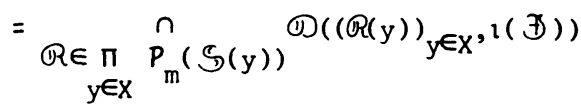

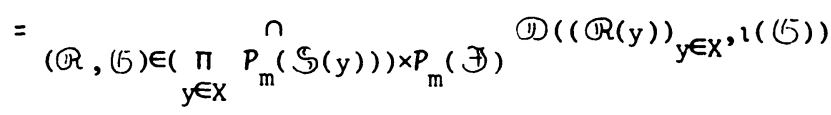

Now applying $(C c)$ on the collection of prime prefilters

$$
\left\{\left(\mathbb{D}\left((\mathbb{R}(\mathrm{y}))_{y \in X}, l(\mathbb{G})\right) \mid\left(\mathbb{R},(\mathfrak{G}) \in\left(\prod_{y \in X} P_{m}(S(y))\right) \times P_{m}(\mathbb{J})\right\}\right.\right.
$$

and the trivial filter on the indexset we obtain

$$
\begin{aligned}
& \lim (1)\left((S(y))_{y \in x}, l(\exists)\right) \\
& \quad\left(R,(5) \in\left(\prod_{y \in x} P_{m}(S(y))\right) \times P_{m}(\Im)\right)^{\lim (1)\left((R(y))_{y \in x}, l((5))\right)}
\end{aligned}
$$

Combining (3.1) and (3.2) we now obtain

$$
\begin{aligned}
\lim & \left((S(y))_{y \in X}, l(\exists)\right) \\
> & \inf _{\mathscr{G} \in P_{m}(\exists)} \lim (5) \\
> & \lim \rightrightarrows .
\end{aligned}
$$

Finally that $\bar{\mu}=\sup _{\mathscr{G} \in \mathbb{P}_{m}(\dot{\mu})} \lim \mathscr{G}$ is an immediate consequence of Proposition 2.10.

4. SUFFICIENCY.

THEOREM 4.1. If $P(X) \rightarrow I^{X}: \exists \rightarrow \lim \rightrightarrows$ is a map which satisfies the properties $(F 1),(F 2),(C 1),(C 2),(C C)$ and (Cd) then there exists a unique fuzzy topology on $X$ such that for each $\oiint \in P(X)$ its limit coincides with $\lim \boldsymbol{B}$.

PROOF. We define the following map

$$
I^{X} \rightarrow I^{X}: \mu \rightarrow \bar{\mu}:=\sup _{\mathfrak{G} \in P_{m}(\dot{\mu})} \lim \mathbb{S} \text {. }
$$


If $\alpha$ is a constant fuzzy set then by. (F1) we have

If $\mu \in I^{X}$ then by (C1) we have

$$
\bar{\alpha}=\sup _{u \text { ultra }} \lim \omega(u) \vee \dot{\alpha}<\alpha .
$$

$$
\begin{aligned}
\bar{\mu} & =\sup _{u \in u(l(\dot{\mu}))} \lim \omega(u) v \dot{\mu} \\
\geqslant & \sup _{\left.\left.x \in \mu^{-1}\right] 0,1\right]} \lim \mu(x) \dot{1}_{x} \\
\geqslant & \sup _{\left.\left.x \in \mu^{-1}\right] 0,1\right]} \mu(x) 1_{x} \\
& =\mu
\end{aligned}
$$

If $\mu, \nu \in I^{X}, \mu \leqslant \nu$ then by (F2) we have

$$
\begin{aligned}
\bar{\mu} & =\sup _{u \in U(l(\dot{\mu}))} \lim \omega(u) v \dot{\mu} \\
& \leqslant \sup _{\operatorname{u\in U}(\imath(\dot{\mu}))} \lim \omega(u) v \dot{v} \\
& \leqslant \sup _{\text {ueu(l( }(\dot{v}))} \lim \omega(u) v \dot{v} \\
& =\bar{v}
\end{aligned}
$$

Further if $\mu, \nu \in I^{X}$ then from this last relation it already follows that $\overline{\mu v \nu}>\bar{\mu} v \bar{\nu}$. On the other hand by Proposition 2.6 we obtain

$$
\begin{aligned}
\overline{\mu v v} & =\sup _{\mathscr{G} \in P_{m}(\mu v v)} \lim \circlearrowleft \\
& \leqslant \sup _{\mathscr{G} \in P_{m}(\dot{\mu})} \lim \circlearrowleft v \sup _{G \in P_{m}(\dot{v})} \lim \circlearrowleft \\
& =\bar{\mu} v \bar{v} .
\end{aligned}
$$

Globally this shows that for any $\mu, \nu \in I^{X}$

$$
\overline{\mu v v}=\bar{\mu} v \bar{v}
$$

Before showing that the map ${ }^{-}$is idempotent we now first define

$$
\lim ^{\prime}: P(X) \rightarrow I^{X}
$$

determined by

$$
\lim \rightrightarrows= \begin{cases}\inf _{\mu \in \exists} \bar{\mu} & \text { if } \exists \text { is prime } \\ \inf _{\mathcal{G} \in P_{m}(\exists)} \lim ^{\prime} \mathscr{G} & \text { otherwise }\end{cases}
$$

ASSERTION. If $\rightarrow$ is prime then $\lim \rightarrow=\lim \exists$.

If $\mu \in \exists$ then clearly $\omega(\imath(\exists)) \vee \dot{\mu} \subset \exists$. Since both are prime, applying (F2) we obtain

$$
\begin{aligned}
\lim \exists & \leqslant \lim \omega(\imath(\exists)) \vee \dot{\mu} \\
& \leqslant \operatorname{u\in u}(l(\dot{\mu}))_{\operatorname{sim} \omega(u) \vee \dot{\mu}}
\end{aligned}
$$

and consequently $\lim \exists \leqslant \lim \rightarrow$ 
Conversely,

$$
\begin{aligned}
& \lim ^{\prime} \exists=\inf _{\mu \in \nexists} \bar{\mu} \\
& =\inf _{\sup } \lim \omega(U) \vee \dot{\mu} \\
& \mu \in \exists \text { u } u \in(i(\mu)) \\
& =\sup \quad \inf \lim \omega(\varphi(\mu)) \vee \dot{\mu} \\
& \varphi \in \prod_{\mu \in \exists} \mathbf{U}(l(\dot{\mu})) \quad \mu \in \exists
\end{aligned}
$$

Now let us fix $\varphi \in \prod_{\mu \in \mathcal{B}} \mathbf{U}(l(\dot{\mu}))$, and consider the family of prime prefilters

$$
\{\omega(\varphi(\mu)) v \dot{\mu} \mid \mu \in \nexists\}
$$

On the indexset $\exists$ we take the filter $A$ generated by the basis

$$
\left\{A_{\mu} \mid \mu \in \exists\right\}
$$

where $A_{\mu}:=\{\nu \in \ni \mid \nu \leqslant \mu\}$.

It is easily seen that now

$$
\text { (7) (1) }\left((\omega(\varphi(\mu)) v \dot{\mu})_{\mu \in \mathcal{B}}, A\right)
$$

By respectively (F2) and (Cc) we then obtain

$$
\begin{aligned}
\lim \exists & \geqslant \lim (1)\left((\omega(\varphi(\mu)) v \dot{\mu})_{\mu \in \mathcal{I}, A)}\right. \\
& \geqslant \inf _{\mu \in \mathcal{I}} \lim \omega(\varphi(\mu)) \vee \dot{\mu}
\end{aligned}
$$

By the arbitrariness of $\varphi$, it now follows from (4.3) and (4.4) that $\lim \rightarrow<\lim .7$. ASSERTION. For any prefilter $\exists$ we have $\lim { }^{\prime} \exists=\lim \exists$.

Applying $(C c)$ on the family of prefilters $P_{m}(\exists)$ and taking hereon the trivial filter we already obtain

On the other hand by (C2) we have

$$
\lim \exists \geqslant \inf _{\left(\zeta \in P_{m}(\exists)\right.} \lim (\zeta .
$$

$$
\begin{aligned}
& \inf _{G \in P_{m}(\exists)} \lim \left(5=\inf _{\operatorname{UaU}(2(\exists))} \lim \omega(U) \vee \exists\right. \\
& \geqslant \inf _{\operatorname{u\in U}(\imath(\exists))} \lim \omega(\imath(\exists)) \vee \rightrightarrows \\
& =\lim \exists \text {. }
\end{aligned}
$$

Consequently by the first assertion

$$
\begin{aligned}
& \lim \rightarrow=\inf _{\left(\zeta \in P_{m}(\exists)\right.} \lim ^{\prime}(\zeta \\
& =\inf _{\circlearrowleft \in P_{m}(\exists)} \lim (5 \\
& =\lim \exists \text {. }
\end{aligned}
$$

From these three assertions we thus conclude that lim satisfies

$$
\lim \exists= \begin{cases}\inf _{\mu \in \exists} \bar{\mu} & \text { if } \exists \text { is prime } \\ \left(\inf _{\mathrm{m}}(\exists)\right. & \text { otherwise }\end{cases}
$$


To conclude the proof of the theorem we shall now show that the map - is idempotent. Let $\mu \in I^{X}$ and $\mathscr{G} \in P_{m}(\dot{\bar{\mu}})$ be fixed.

Now remark that the proof of Proposition 2.12 only uses the fact that closure and limit in a fuzzy topological space fulfil (4.1), (4.2) and (4.5). Since we have already shown that ${ }^{-}$indeed fulfils $(4.1),(4.2)$ and $(4.5)$, by means of a perfectly analogous proof, we can now ascertain that for each $y \in X$ there exists $\mathcal{S}(y) \in P_{m}(\dot{\mu})$ such that $\lim S(y)(y)=\bar{\mu}(y)$.

Consider this selection $(S(y))_{y \in X}$, then clearly $\rho_{\mathcal{S}}=\bar{\mu}$ and so $\rho_{\mathcal{S}} \in G$. Applying (Cd) we now obtain for (1) := (1) $\left((S(y))_{y \in X}, l(\mathbb{S})\right)$ that

$$
\lim (1) \geqslant \lim (5
$$

which together with the facts that $\mu \in$ (D) -which is obvious since $\mu \in \mathcal{S}(y)$ for each $y \in x$ - that $(0)$ is prime -by Proposition $2.7 .4^{\circ}$ - and upon applying (5) implies that

$$
\bar{\mu} \geqslant \lim 6
$$

By the arbitrariness of $\mathscr{G} \in P_{\mathrm{m}}(\dot{\bar{\mu}})$ we thus obtain

$$
\bar{\mu} \geqslant \sup _{\mathscr{\zeta} \in P_{m}(\dot{\bar{\mu}})} \lim \mathscr{E}=\overline{\bar{\mu}} .
$$

In all we have thus shown that - is the fuzzy closure operator associated with a fuzzy topology. That the limit in this fuzzy topology coincides with the map lim is nothing else than (5) while uniqueness of the fuzzy topology is evident by construction. 5. PRTME VERSIUN.

Both in Theorem 3.1 and Theorem 4.1 prime prefilters play a crucial role in proofs. The question therefore poses itself whether it is not sufficient to consider (F1), (F2), (C2), ( CC), (Cd) restricted to prime prefilters, i.e. to consider what we shall call prime versions of these properties. Remark that obviously (C1) is its own prime version. In order to answer this question we now define the following set of axioms.

(F1p) For any prime prefilter $\exists$ : $\lim \exists<c(\exists)$

(F2p) For any prime prefilters $\exists, \mathcal{H}: \exists \subset \exists \Rightarrow \lim \mathbb{H}<\lim \exists$

(c2p) For any prefilter $\exists:\left(5 \in P_{m}(\exists) \Rightarrow \lim \exists \leqslant \lim (5\right.$

( Ccp) For any collection of prime prefilters $\left(\exists_{j}\right)_{j \in J}$ and any filter $A$ on $J$ :

$$
\lim (1)\left(\left(\exists_{j}\right)_{j \in J}, A\right) \geqslant \inf _{j \in J} \lim \exists_{j}
$$

(Cdp) For any prime prefilter $\exists$ and any selection of prime prefilters $(\mathcal{S}(y))_{y \in X}$ such that $\rho_{S} \in \exists: \lim (1)\left(\left(S(y)_{y \in X}, l(\exists)\right)>\lim \rightrightarrows\right.$

PROPOSITION. The following implications hold :

$1^{\circ}(\mathrm{C} 2 \mathrm{p})$ implies $(\mathrm{F} 1) \Leftrightarrow(\mathrm{F} 1 \mathrm{p})$

$2^{\circ}(\mathrm{C} 2 \mathrm{p})+(\mathrm{Ccp})$ implies $(\mathrm{F} 2) \Leftrightarrow(\mathrm{F} 2 \mathrm{p})$

$3^{\circ}(\mathrm{Ccp})$ implies $(\mathrm{C} 2) \Leftrightarrow(\mathrm{C} 2 \mathrm{p})$

$4 \circ(\mathrm{C} 2 \mathrm{p})$ implies $(\mathrm{Cc}) \Leftrightarrow(\mathrm{Ccp})$

$5^{\circ}(\mathrm{C} 2 \mathrm{p})+(\mathrm{ccp})$ implies $(\mathrm{cd}) \Leftrightarrow(\mathrm{cdp})$.

PROOF. $1^{\circ}$ This goes the same as the proof of (F1) in Theorem 3.1.

$2^{\circ}$ Let $\exists$ be any prefilter. Since $\exists=\underset{\left(\mathcal{G} \in P_{\mathrm{m}}(\exists)\right.}{(G)}$ we obtain by (Ccp) applied to the trivial filter on $P_{m}(\exists)$ that

$$
\lim \exists \geqslant \inf _{\left(\mathfrak{G} \in P_{m}(\exists)\right.} \lim \mathscr{G}
$$


and consequently together with $(\mathrm{C} 2 \mathrm{p})$ that

$$
\lim \exists=\inf _{\mathscr{b} \in P_{m}(\Im)} \lim (5
$$

The rest of the proof now consists of repeating verbatim the demonstration of (F2) in Theorem 3.1.

$3^{\circ}$ Let $\rightarrow$ and $\mathcal{H} \in \mathcal{2}_{m}(\exists)$ be arbitrary. First by repeating the first part of the proof of $2^{\circ}$ we note that $(5.1)$ holds. Second by (5.1) and Proposition 2.4 we obtain :

$$
\begin{aligned}
& \lim \rightarrow=\inf _{\mathfrak{S} \in P_{m}(\mathcal{B})} \lim \mathbb{G} \\
& \leqslant \inf _{\mathfrak{G} \in P_{m}(\mathcal{H}())} \lim \mathbb{L} \\
& =\lim 3
\end{aligned}
$$

$4^{\circ}$ This is completely analogous to the proof of the general case of $(C c)$ in Theorem 3.1 .

$5^{\circ}$ This in turn is completely analogous to the proof of the second assertion in the proof of ( $C d$ ) also in Theorem 3.1.

From this proposition and Theorem 4.1 we now immediately obtain the following strengthening of the latter.

THEOREM 5.1. If $P(X) \rightarrow I^{X}: \exists \rightarrow \lim \rightrightarrows$ is a map which satisfies the properties $(F 1 p),(F 2 p),(C 1),(C 2 p),(C C p)$ and (Cdp) then there exists a unique fuzzy topology on $X$ such that for each $\Im \in P(X)$ its limit coincides with lim $¥$.

\section{REFERENCES}

1. BILLINGSLEY, P. Convergence of probability measures, Johri Wiley \& Sons, New York, 1968.

2. BIRKHOFF, G. Moore-Smith convergence in general topology, Ann. Math. (2) 38 (1937), 39-56.

3. BOURBAKI, N. Topologie générale, chapt. 1 et 2, Hermann, Paris, 1965.

4. CARTAN, H. Théorie des filtres, C.R. Acad. Sci. Paris 205 (1937), 595-598.

5. COOK, C.H. and FISHER, H.R. Regular convergence spaces, Math. Ann. 174 (1967), $1-7$.

6. KENT, D.C., RICHARDSON, G.D. Compactification of convergence spaces, Internat. J. Math. and Math. Sci. 2 2 (1979), 345-308.

7. KOWALSKY, H. Limesraüme und Komplettierung, Math. Nachr. 12 (1954), 301-340.

8. LOWEN-COLEBUNDERS, E. An internal and an external characterization of convergence spaces in which adherences of filters are closed, Proc. Amer. Math. Soc. 72 (1978), 205-210.

9. LOWEN-COLEBUNDERS, E. On the regularity of the Kowalsky completion, Canad. J. Math. 36 (1984), 58-70.

10. LOWEN, R. Fuzzy topological spaces and fuzzy compactness, J. Math. Anal. Appl. 56 (1976), 621-633.

11. LOWEN, R. Convergence in fuzzy topological spaces, General Topology Appl. 10 (1979), 147-160.

12. LOWEN, R. IX, the hyperspace of fuzzy sets, a natural non-topological fuzzy topological space, Trans. Amer. Math. Soc. 278 (1983), 547-564.

13. LOWEN, R. Hyperspaces of fuzzy sets, Fuzzy Sets and Systems $\underline{9}$ (1983), 287-311.

$1 \%$ LOWEN, R., WUYTS, P. On completeness, compactness and precompactness in fuzzy uniform spaces, J. Math. Anal. Appl. 92 (1983), 342-371. 
15. LOWEN, R. On the existence of natural fuzzy topologies on spaces of probability measures, Math. Nachr. 115 (1984), 33-57.

16. LOWEN, R. The order aspect of the fuzzy real line, Manuscripta Math. 39 (1985), 293-309.

17. LOWEN, R. Metric spaces viewed as fuzzy topological spaces induced by Lipschitz functions, Math. Nachr. 120 (1985), 249-265.

18. MICHAEL, E. Topologies on spaces of subsets, Trans. Amer. Math. Soc. 71 (1951), 152-182.

19. REED, E. Completions of uniform convergence spaces, Math. Ann. 194 (1971), 83-108. 


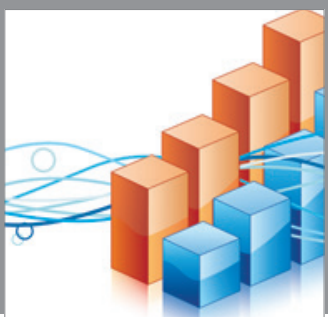

Advances in

Operations Research

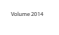

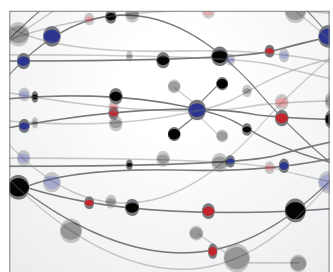

\section{The Scientific} World Journal
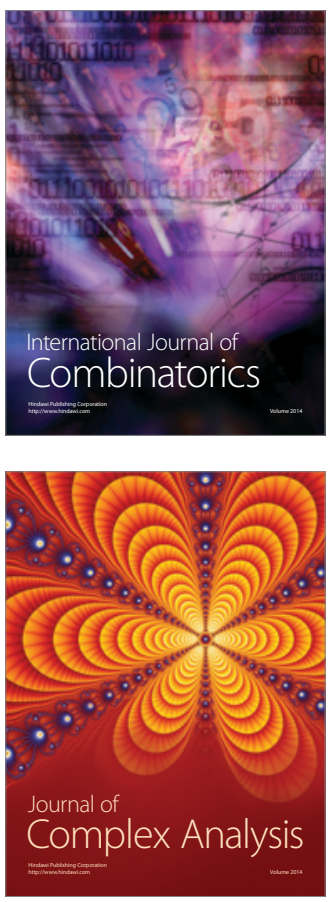

International Journal of

Mathematics and

Mathematical

Sciences
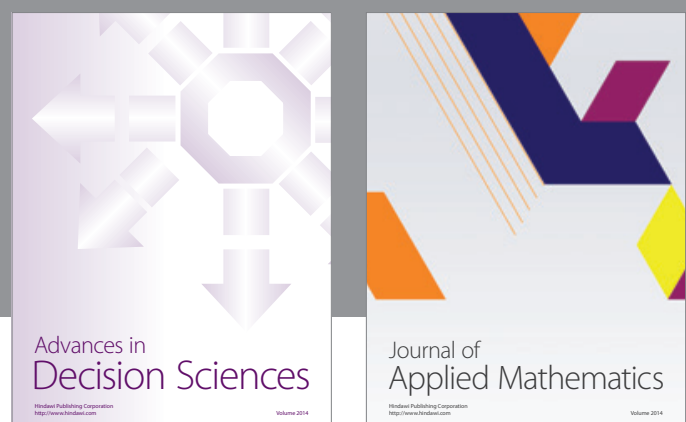

Journal of

Applied Mathematics
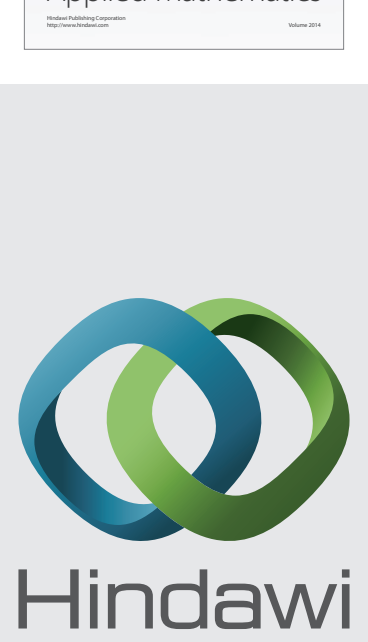

Submit your manuscripts at http://www.hindawi.com
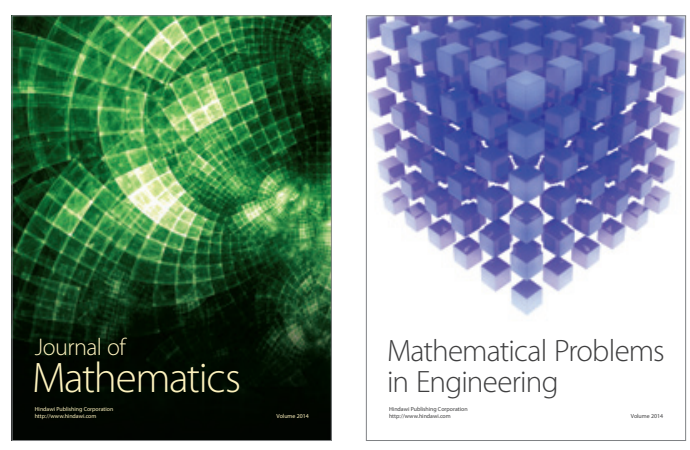

Mathematical Problems in Engineering
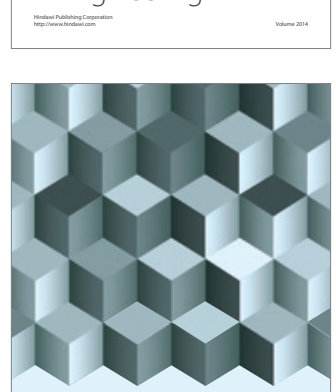

Journal of

Function Spaces
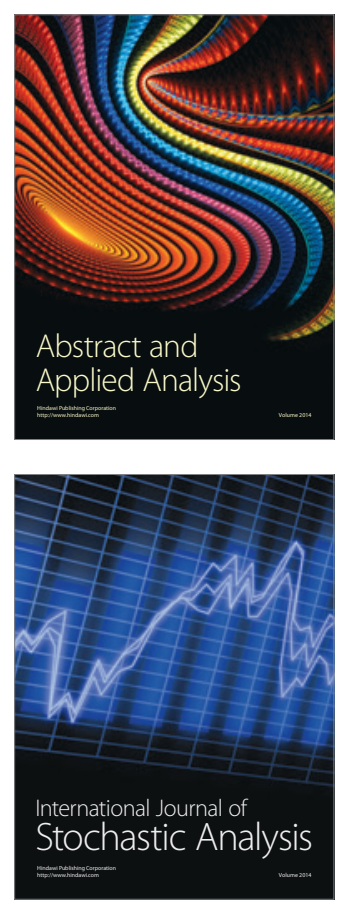

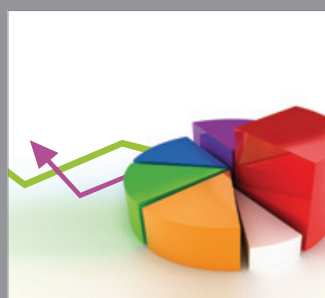

ournal of

Probability and Statistics

Promensencen
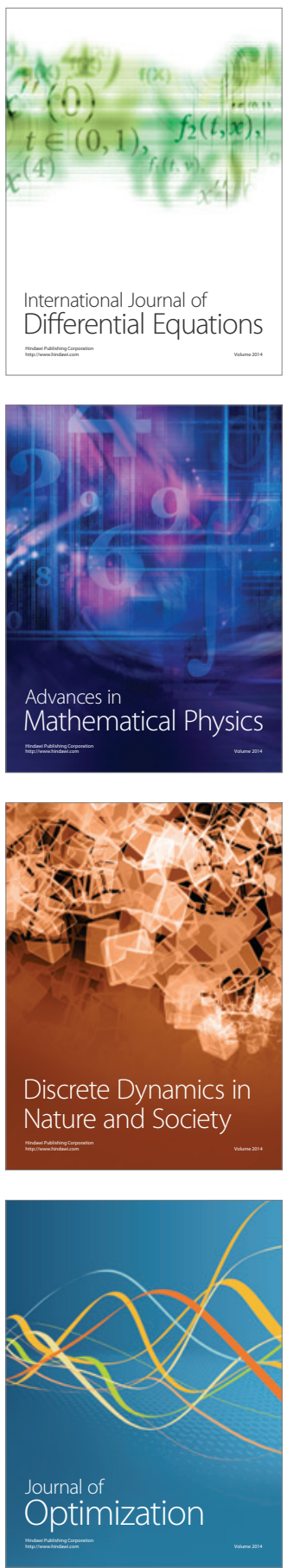